Int. J. Dev. Biol. 53: 683-692 (2009)

doi: $10.1387 /$ ijdb.092936rh

\title{
The emergence of patterning in life's origin and evolution
}

\author{
ROBERT M. HAZEN* \\ Geophysical Laboratory, Carnegie Institution, Washington, USA
}

\begin{abstract}
Three principles guide natural pattern formation in both biological and non-living systems: (1) patterns form from interactions of numerous individual particles, or "agents," such as sand grains, molecules, cells or organisms; (2) assemblages of agents can adopt combinatorially large numbers of different configurations; (3) observed patterns emerge through the selection of highly functional configurations. These three principles apply to numerous natural processes, including the origin of life and its subsequent evolution. The formalism of "functional information," which relates the information content of a complex system to its degree of function, provides a quantitative approach to modeling the origin and evolution of patterning in living and nonliving systems.
\end{abstract}

KEY WORDS: pattern formation, biocomplexity, emergent systems

Complex patterning is a hallmark of biological systems at every scale, from molecules to cells to organisms to ecosystems. Life's triumph of order over chaos is the epitome of the more general natural phenomenon of emergent systems, in which numerous components or "agents" respond to their local environments, thus interacting to produce patterns or behaviors not characteristic of individual agents. Such patterning arises at every scale of the physical universe, for example in the behaviors of sand dunes (Bagnold 1988; Hansen et al. 2001), turbulent fluids (Frisch 1995), and spiral galaxies (Carlberg 1992). Complex behaviors also emerge in a range of artificial symbolic contexts, including artificial life programs (Adami 1995), cellular automata (Wolfram 2002), and models of market economies (Holland 1995). However, while such symbolic systems can provide insights into mechanisms of physical pattern formation, this article will focus exclusively on natural systems of tangible agents.

Life, with its novel patternings and collective behaviors, is the quintessential emergent complex phenomenon. Life's ancient origin has been modeled as a sequence of emergent events that, step by step, transformed the lifeless geochemical world of oceans, atmosphere and rocks into a living planet (De Duve 1995; Morowitz 2002; Hazen 2005). These sequential processes began with the synthesis of key biomolecules, including amino acids, sugars, lipids and bases, in a variety of prebiotic environments (Miller 1953; Miller and Urey 1959; Heinen and Lauwers 1996; Bernstein etal. 1999; Ehrenfreund and Charnley 2000; Cody etal. 2004). Then, a subset of those diverse molecules had to be selected and concentrated, for example through a self-organizing process (Deamer and Pashley 1989; Dworkin et al. 2001; Hazen and Deamer 2007) or by adsorption on mineral surfaces (Bernal 1951; Ferris 1999; Hazen 2006). Ultimately, the key step in the ancient transition from geochemistry to biochemistry was the emergence of the first self-replicating molecular system (Gilbert 1986; Orgel 1986; Morowitz 1992; Kauffman 1993), and the consequent triggering of rapid evolution through competition and natural selection (Sagan 1961; De Duve 2005).

The origin of cellular life and its rapid radiation across the globe triggered an explosion of new collective biological forms, functions and behaviors. Numerous examples of novel pattern formation in biological systems have been modeled in terms of emergence, including the aggregation of social amoebas, the functioning of neural networks, the formation of spotted or striped skin pigmentation, the swarming behavior of ants, and many other phenomena (Jung etal. 1998; Allen etal. 1998; Solé and Goodwin 2000; Camazine et al. 2001; Avery 2003; Maini et al. 2006; Sick et al. 2006; Deamer and Evans 2006; Borman 2006).

The study of emergent patterning is especially pertinent to the field of development biology, as represented by the collected articles of this volume. Each cell in a developing embryo responds principally to its immediate physical and chemical environment in space and time, for example through mechanical interactions, chemical signaling, and the sequential expression of different genes. Yet these local cellular interactions lead to the remarkable collective morphological diversity of distinctive organs and tissues, from hearts and eyes to bones and teeth (Lawrence 1992; Piekny and Mains 2003; Wu et al. 2004; Nüsslein-Volhard 2006). Furthermore, diverse cellular morphogens and signaling pathways provide opportunities for combinatorially vast numbers of

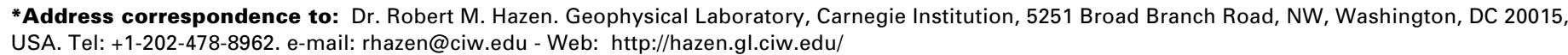

Published online: 29 May 2009.

ISSN: Online 1696-3547, Print 0214-6282

(C) 2009 UBC Press

Printed in Spain 

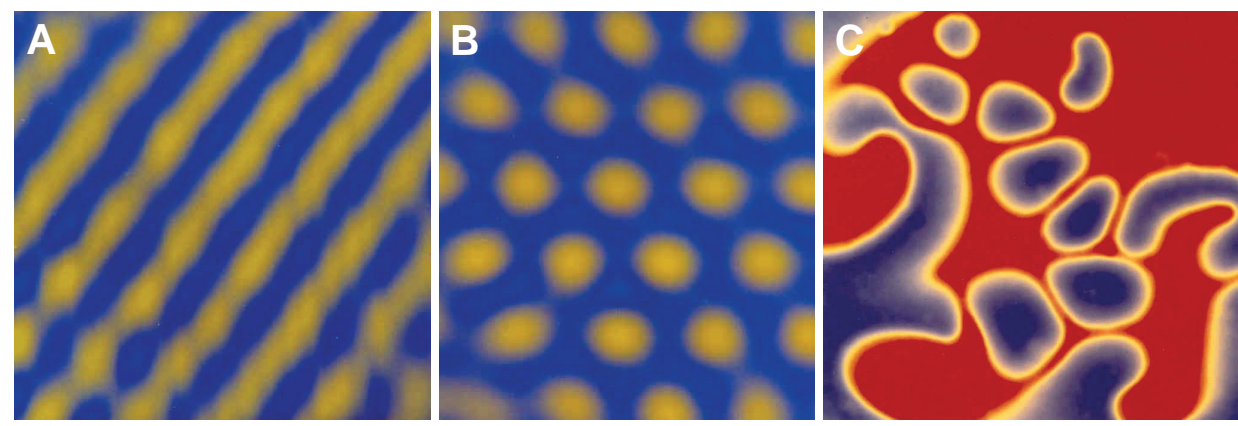

Fig. 1. (A) Linear, (B) spot and (C) globular patterns formed in systems of two reacting chemicals (Ouyang and Swinney 1991). Photographs courtesy of H. L. Swinney.

alternative interactions, and a corresponding richness in evolutionary potential under selective pressures.

The field of emergent complexity thus provides a powerful framework for understanding the origin and evolution of patterning in any physical context, including the development of biological architecture. The objective of this article is to define general principles by which patterns form in complex systems, and to propose a universal formalism for quantifying the information content necessary for the emergence of such systems.

Studies of patterning in both living and nonliving natural systems point to three general principles - factors related to (1) the interaction of agents, (2) the diversity of configurations, and (3) the degree of function - that influence the emergence of pattern formation. In the following sections we review these three principles.

\section{Agents and their interactions}

\section{Principle 1: patterns form as a consequence of the interac- tions of numerous agents \\ The nature of agents}

Patterns emerge when numerous individual agents interact (e.g., Holland 1995). Thus, for example, patterning in living systems may arise from interactions of small molecules to form membranes, biopolymers to form muscle fibers, cells to form multi-cellular organisms, or multi-cellular organisms to form colonies. Patterns in nonliving systems, similarly, are observed to emerge from physical, chemical, and gravitational interactions of such diverse agents as water molecules, sand grains, automobiles or stars. (Note also that agents in symbolic systems, including sequences of letters, numbers, musical notes or machine commands, play analogous roles by interacting under sets of mathematical or computational selection rules or protocols.) In every case numerous agents interact to produce patterns and behaviors not associated with the traits of individual agents: a single sand grain cannot form a ripple, a single neuron cannot be conscious, nor can a single ant display the complex collective behaviors of an ant colony.

In some systems all agents are identical to each other. Thus, patterns may emerge in interacting systems with pure water molecules, uniform microspheres, or identical automobiles. On the other hand, complex systems often display a variety of interacting agents. Sand grains provide an instructive example, because sediment grains may be relatively uniform in size, shape and composition (a situation found in the well-sorted sand dunes and ripples at many beaches), or they may vary significantly in size, shape and density (as observed in the heterogeneous sediments of deltaic deposits downstream of rapidly eroding mountains). Note that a greater variety of sediment grains leads to greater opportunities for patterning, for example in the dynamic size-sorting of graded bedding (Pettijohn 1957) or the density separation of gold grains into rich placer ore deposits (Park and MacDiarmid 1964).

The case of developing biological systems is especially intriguing (and complicated), in part because agents can be analyzed at several scales, including small molecules, biopolymers, cells and cell clusters. [At the smallest scale, living systems have also been discussed in terms of quanta (Morowitz 1982).] This hierarchical aspect of embryogenesis may lead to confusion regarding the number of levels of information that must be integrated when studying development. Nevertheless, whatever set of agents is considered, the collective behavior of the system arises from the response of each agent to its local environment.

\section{Selection rules}

Given a collection of interacting agents, what are the immediate mechanisms of pattern formation? In many systems complex patterns are known to arise from a few simple selection rules or protocols that are followed by each agent. Thus, for example, the formation of sand patterning, including a rich variety of ripples and dunes, results from the net additive effect of three kinds of forces, each of which represents a selection rule that operates on each grain. The vertical gravitational force on each grain and the contact forces from adjacent grains tend to keep a grain in place, while the horizontal force of moving wind or water tends to move grains laterally (Bagnold 1988). Collective patterns emerge as each individual grain responds only to these local forces.

Selection rules at several levels of organization are also observed in biological systems (Doyle and Csete 2007). The seemingly random yet remarkably synchronized schooling patterns of fish or flocking behavior of birds provide dramatic examples of selection rules. Cohesion is achieved when each individual in the group responds to the positive feedback of staying close to its mates, balanced by the negative feedback of avoiding collisions (Camazine et al. 2001; Fedder 2007; Parrish and Hamner, 1997). Thus individual birds or fish need not monitor the actions of the entire system, nor is it necessary for any one individual to act as leader.

Simple rules also describe the behaviors of ant and termite colonies. The coordinated behavior of ant swarm raids and the complex architecture of ventilated termite mounds result from responses of numerous individuals to a small number of local chemical and physical stimuli (Wilson and Hölldobler 1988; Franks etal. 1992; Franks and Deneubourg 1997; Camazine et al. 2001). Again, there does not appear to be any one leader, architect or blueprint.

The role of selection rules in pattern formation underscores the importance of information transfer among individuals and their 
environments. Each agent obtains information from its environment through interactions. The contact forces between sand grains, the visual proximity of two birds, the pheromone trails of ants, or the gravitational fields between stars represent modes of information transfer that ultimately lead to patterning. Each interaction is thus equivalent to the transfer of information from one agent to another.

\section{The role of gradients}

Patterns, by definition, represent deviations from a homogeneous equilibrium state. In both living and non-living physical systems, pattern formation is a dynamic, non-equilibrium phenomenon of self-organization that arises from the interaction of agents under the influence of a gradient (e.g., in density, chemical concentration, temperature, velocity, or electrical or gravitational potential). Gradients have been cited as critical to pattern formation because patterns arise spontaneously as energy is dissipated into the environment against various constraints, such as chemical or thermal diffusion, in accord with the second law of thermodynamics (Nicolis and Prigogine 1989; Ouyang and Swinney 1991; Petrov et al. 1997; Swinney 2000; Hoyle 2006). Indeed, in such "dissipative systems" patterns may be analyzed as being arrangements of agents that arise from the tendency of agents to attain equilibrium at a local scale, while heterogeneities occur at a much larger scale.

A system near equilibrium tends to respond to perturbations through the rapid decay of that perturbation. Thus, small local excursions in the temperature, velocity or chemical composition of a near-homogeneous fluid will tend to even out and return the system to a homogeneous (i.e., high symmetry) state. However, if such a system is perturbed beyond a critical value, for example by heating from below or by the continuous injection of a dye, the perturbation may grow and produce patterns that break the symmetry of the near-equilibrium state (Swinney 2000). Such patterning arises as the most efficient means to re-attain equilibrium (i.e., to eliminate the local gradient as rapidly as possible).

Consider, for example, a shallow pan of water uniformly heated from below (Koschmeider 1981; Nicholis and Prigogine 1989). As long as the thermal gradient between the water's lower and upper surface remains below a critical value, the liquid will retain its homogeneous state (i.e., it will adopt the symmetry of the boundary conditions). However, at gradients above the critical value the shallow water is observed to divide into numerous small convection cells, called Bénard cells, which break the symmetry of the boundary conditions to form a pattern (Swinney 2000). These convection cells are able to distribute thermal energy (and thus reduce the thermal gradient) more efficiently than heat dissipation in the homogeneous fluid.

An analogous situation may obtain in chemical systems. Below a critical concentration gradient, perturbations can be damped by diffusion alone. However, above that critical chemical gradient new patterns, such those formed by the well known BelousovZhabotinski or BZ reactions, arise (Vidal and Pacault 1984; Field and Burger 1985; Nicolis and Prigogine 1989). Depending on the gradients and reaction rates, a variety of spiral, branching and oscillatory patterns emerge (Table 1; Figures 1-6). Strikingly similar patterns, also the consequence of chemical signaling, may emerge in nutrient-limited cultures of bacteria (Ben-Jacob et al. 1994; Ko etal. 1994). In like manner, compositional gradients may promote a host of phenomena in developing multi-cellular organisms, including invertebrate segmentation, vertebrate somite formation, and patterning in skin and mollusk pigmentation (Turing 1952; Meinhardt 1995; Palmeirin etal. 1998; Solé and Goodwin 2000).

\section{Internal versus external controls on interactions}

Pattern formation requires the transfer of information from the environment to agents. A common feature of emergent systems is that agents obtain their information exclusively from their local environments, as opposed to receiving instructions from a leader or blueprint. Agents may interact directly, as in two touching sand grains, or indirectly if one agent alters the environment in such a way as to affect other agents (Figures 1-6). Nevertheless, agents need not be guided by a global information system.

Numerous familiar systems of interacting agents do not conform exactly to this model because their behavior is dictated, at least in part, by information from an external supervisor or master program. In these cases individuals do not respond exclusively to local stimuli. The interactions of musicians illustrate the continuum of such external controls.

Consider first a football marching band that employs numerous individual musicians to spell out a school letter or logo. As in emergent systems, the observed pattern is formed by many individual agents, but in this case each individual acts in a predetermined manner with an assigned place and programmed movements. The marchers may incorporate minor adjustments in their positions and their playing in response to the small perturbations of adjacent individuals, but the overall pattern formation is dictated by an external blueprint.

In a symphony orchestra, similarly, the conductor and musical score together dictate the action of each player; however, each symphony musician has a modest degree of individual leeway in performance style. Indeed, such emergent behavior, with each musician responding in part to immediate local influences of fellow instrumentalists, provides the spontaneity of a live orchestral performance. Increasing emergent behavior arises in a jazz ensemble, where all musicians follow the basic guidelines of a "lead sheet," but are free to interact with exuberant spontaneity.

The example of music illustrates three important aspects of the interactions that lead to pattern formation that are also relevant to
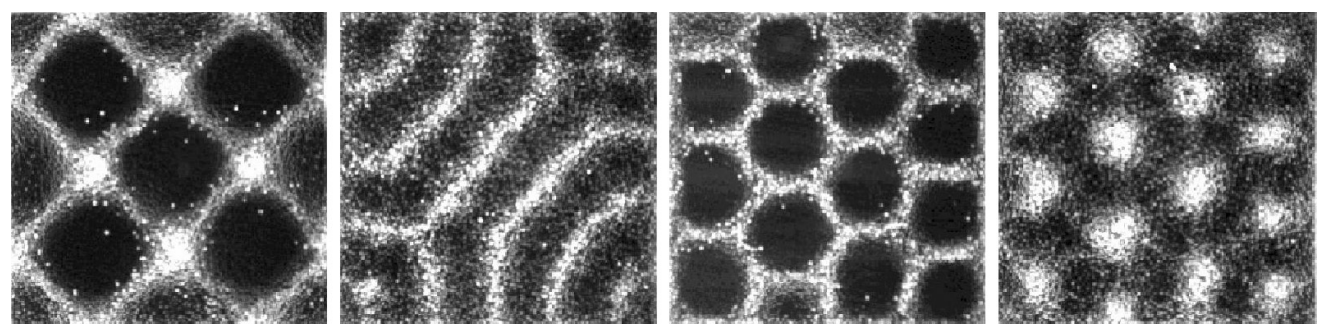

Fig. 2. Patterns formed in sand on a vibrating table. Different patterns, including squares, lines, hexagons and periodic dots, arise at different vibration frequencies (Swinney 2000). Photographs courtesy of H. L. Swinney. 
biology. (1) In music, as in biology, patterns typically emerge from interactions simultaneously at more than one level. Thus, the music we hear results from at least two levels of organization - from the sequential superposition of numerous individual pitches and timbres (sound waves that form a melody, for example), and also by the collective efforts of numerous individual musicians. (2) Interactions are governed by selection rules or protocols. Thus, the arrangements of musical pitches of successful musical compositions are guided rules of harmony and counterpoint. And (3), there is not always a strict dichotomy between internal and external controls on interactions; both often come into play during a musical performance, as they will in some instances of biological pattern formation. What is clear is that many patterns in biological systems (e.g., Table 1) can form strictly by local interactions, in parallel to pattern formation in non-living systems with no obvious external control.

\section{The diversity of configurations}

\section{Principle \#2: interacting agents can adopt combinatorially large numbers of distinct configurations}

We have seen that emergent patterning arises from the interaction of agents. A key to pattern formation is that assemblages of agents have the potential to adopt combinatorially large numbers of different configurations. The diversity of possible configurations depends on three principal factors: (1) the number of interacting agents, (2) the number of different kinds of agents, and (3) the nature of interactions among agents.

Consider the example of RNA oligomers. For a standard 3'5 ' linked RNA strand composed of a sequence of four nucleotides, the number of possible different sequence configurations, $N$, depends only on the number of nucleotides, $n: N=4^{n}$. If, however, we add $x$ non-conventional bases (i.e., a greater diversity of agents), then the number of sequence configurations increases to $N=(4+x)^{n}$. And if we allow both 3'-5' and 2'5' linkages (i.e., a greater variety of interactions between agents), then the number of sequence configurations further increases to $N=(4+x)^{2 n}$.

In many systems of interest the number of configurations, though large, is finite. For example, a sequence of 100 letters in the standard English alphabet has $26^{100}$ possible configurations. Similarly, a 100-nucleotide strand of RNA has $4^{100}$ different configurations. Though these numbers of different configurations are large, these systems are of modest complexity compared to many everyday examples. Consider, for example, the number of different possible neural network configurations of a human brain with $10^{10}$ neurons, each connected to hundreds of other neurons.

The nature of interactions is especially important in deter-
TABLE 1

\section{COMMON REGULAR SPACIOTEMPORAL MODES OF PATTERNING}

\begin{tabular}{lll} 
Pattern & Examples & Reference \\
\hline Uniform striping or banding & Sand ripples (steady flow) & Bagnold (1988) \\
& Thin-layer convection rolls & Solé \& Goodwin (2000) \\
& Stationary chemical patterning & Ouyang \& Swinney (1991) \\
& Embryo segmentation & Lawrence (1992) \\
Bifurcating stripes & Sand ripples (oscillating flow) & Hansen et al. (2001) \\
& Zebra stripes & Murray (1981) \\
Irregular hexagons & Bénard cells (heated water or oil) & Nicolis \& Prigogine (1989) \\
& Giraffe pigmentation & Koch \& Meinhardt (1994) \\
& Tilapia (fish) nest distributions & Barlow (1974) \\
Squares & Vertically oscillated liquid/spheres & Swinney (2000) \\
Quasiperiodic spots & Stationary chemical patterning & Ouyang \& Swinney (1991) \\
& Shell pigmentation & Meinhardt (1995) \\
& Hair follicle distribution & Sick et al. (2006) \\
Triangular waves & Shell pigmentation & Meinhardt (1995) \\
Spiral defect chaos & Rayleigh-Bénard convection & Solé \& Goodwin (2000) \\
& Belousov-Zhabotinsky reactions & Nettesheim et al. (1993) \\
& Aggregating starving amoebas & Höfer et al. (1995) \\
& Predator-prey spatial distribution & Solé et al. (1992) \\
Branching & Lightning and Streams & Arrayas et al. (2002) \\
& Respiratory, circulatory \& neural & Metzger \& Krasnow (1999 \\
& Mammary glands & Widelitz et al. (2007) \\
& Army ant swarm raid paths & Deneubourg et al. (1989) \\
Circular rings & Patterned arctic ground & Kessler \& Werner (2003) \\
& Leptothorax (ant) wall building & Franks et al. (1992) \\
& Ringworm infections & Deacon (2006) \\
Quasipatterns & Faraday wave experiments & Edwards \& Fauve (1993) \\
& Ni-Cr quasicrystals & Shechtman et al. (1984) \\
\hline & & \\
& &
\end{tabular}

Each of these modes of patterning can arise from gradients, for example in chemical concentration, temperature or velocity. For a mathematical treatment of the formation of these patterns see Hoyle (2006)

mining the number of possible system configurations. In the case of sand grains, all interactions involve direct physical contact with adjacent grains (a number that will rarely exceed 7 or 8 simultaneous contacts), in combination with moving wind or water (Bagnold 1988). In RNA or proteins, by contrast, interactions include both primary structures (the one-dimensional sequence of nucleotides or amino acids, respectively), and the complex secondary and tertiary interactions characteristic of folded macromolecules. In many social organisms, including colonial insects and social amoebas, residual pheromone signals of varying strengths create complex networks of chemical gradients. And in neural networks, numerous interconnections of variable electrical potential among neurons create large numbers of different possible configurations.

A central challenge in understanding developmental biological processes in terms of emergence lies in the combinatorially vast numbers of possible configurations, which arise from the three-dimensional and time-dependent complexity of inter-and intracellular interactions. Not only does each cell (or cell cluster) respond to a variety of local mechanical and chemical signals, but cellular agents also have the distinctive ability to generate new agents through mitosis (or to eliminate agents

Fig. 3. Sand ripple patterns, photographed in oblique light, form in a shallow wave tank (from Hansen et al. 2001). Uniform water oscillations (left image) result in regular linear ripples, whereas variations in wave frequency or amplitude (increasing left to right) result in sinuosity, bifurcation and braiding of ripples. Photographs courtesy of J. L. Hansen. 
through apoptosis). Thus, all three factors - the number of agents, the diversity of agents, and the interactions among agents - contribute to the extreme complexity of developing biological systems.

\section{Patterns and their degree of function}

\section{Principle \#3: observed patterns arise through the selection of functional configurations}

All emergent complex systems arise from interactions among numerous agents, but what factors lead to the appearance of one configuration in preference to another? Why do some patterns tend to form, while others do not? We suggest that patterns typically emerge as a result of selective pressures on a variety of configurations of varying degrees of function. In both living and nonliving systems, therefore, observed patterns may represent the small subset of highly functional configurations.

All complex systems have the ability to do something - to accomplish one or more functions. The function of some emergent systems is obvious: a sequence of letters communicates a specific idea, a computer algorithm performs a specific computation, and an enzyme catalyzes at least one specific bioreaction. Less obvious are the functions of patterned systems of many interacting inanimate particles such as molecules, sand grains or stars, but these systems may also be described quantitatively in terms of function, for example in terms of their structural stability, their ability to dissipate energy, or their ability to maximize entropy production through patterning (e.g., Bertalanffy 1968; Nicolis and Prigogine,1989; Swenson and Turvey 1991; Emanuel 2006). Indeed, this type of self-organizing, entropy-driven pattern formation may play a key role in some localized developmental events in embryos, such as the formation of branching patterns, stripes and dots, and oscillations (e.g., Turing 1952; Lechleiter et al. 1991; Maini et al. 2006; Sick et al. 2006).

Living systems often display multiple essential functions, including the abilities to harvest nutrients and energy, adapt to changes in environment, avoid predation and produce viable offspring (Allen et al. 1998; Ayala 1999; McShea 2000). Consequently, quantification of the degree of function of a multi-cellular organism is challenging. However, the function of specific developing biological structures, such as an enzyme, an organ, or a beak, may be amenable to more rigorous functional analysis.

In the following section we review the concept of "functional information," which provides a measure of the information (in bits) necessary to achieve a specified degree of function. This consideration of complexity in terms of the function of a system, as opposed to some intrinsic measure of its patterning or structural intricacy, distinguishes our treatment from many previous efforts (Gell-Mann 1995; Bell 1997; Kåhre 2002; Gell-Mann and Lloyd 2003; Adami 2003; Von Baeyer 2003; Shalizi 2006).

\section{Functional information}

Given the ubiquity and diversity of emergent systems and pattern formation, it has proven desirable to quantify the characteristics of emergent complex systems, and to identify factors that might promote complexity in evolving systems. The formalism of "functional information" provides a quantitative approach to modeling the origin and evolution of biological patterning, as well as

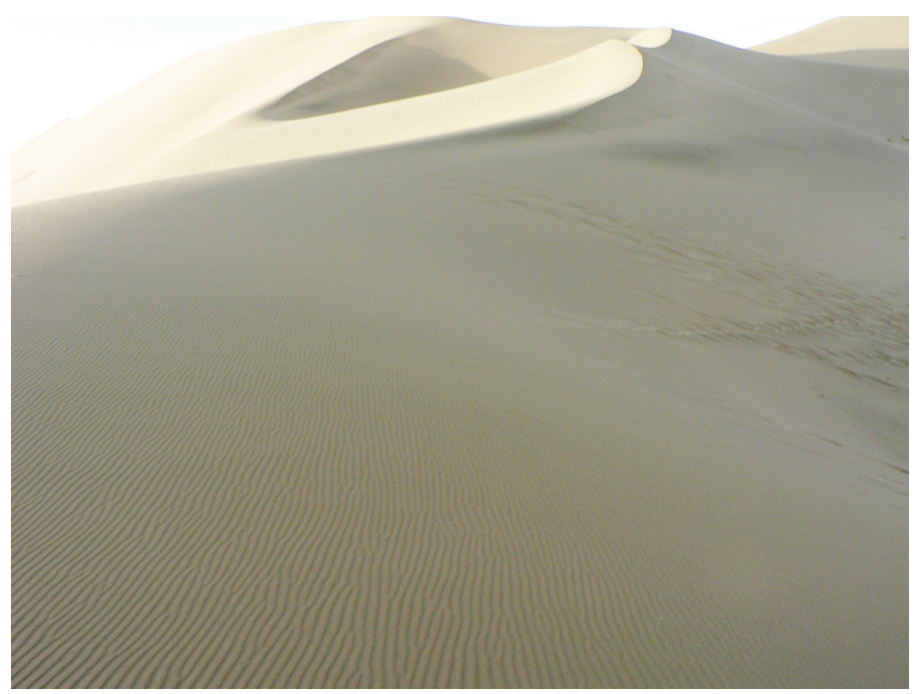

Fig. 4. Bifurcation and braiding of sand ripples on the flanks of a sand dune. Photograph courtesy of C.-M. Chuong.

patterned behavior in other physical and symbolic systems (Szostak 2003; Carothers et al. 2004; Hazen et al. 2007).

We quantify the complexity of a system that can adopt numerous different configurations in terms of the information (in bits) necessary to achieve a specified degree of function. The formalism applies equally to symbolic systems (letter sequences, computer code), nonliving physical systems (sand configurations, spiral galaxies), and living systems (RNA aptamers, colonial insects). Note that all of these examples share the three critical characteristics of emergent systems: (1) they consist of numerous interacting agents, (2) the agents can combine in a combinatorially large number of different configurations, and (3) some configurations display functions that are not characteristic of the individual agents.

Analysis of such emergent systems sheds light on questions related to the close connection between information content and function, including complex functional patterning in evolving biological systems. For example, how much information is required to encode a biological structure, such as a tooth or beak with a specific function? Are there multiple distinct solutions to achieving a specific degree of function and how are such solutions distributed in configuration space? How much additional information is required to attain a specified improvement in function? What environmental factors might lead to new or enhanced modes of patterning?

Szostak and coworkers (Szostak 2003; Carothers et al. 2004) introduced functional information as a measure of complexity in information-rich biopolymers, such as RNA aptamers (RNA structures that bind a target molecule), in contrast to prior formalisms based on genomic, sequence, or algorithmic information (e.g., Adami 2003; Lenski et al. 2003). The concept was subsequently expanded to other emergent systems, including letter sequences and artificial life genomes (Hazen et al. 2007).

\section{Functional information as a measure of system complexity}

Emergent systems of interacting agents can be described in terms of their potential to accomplish one or more quantifiable functions. Consider the system of all possible 100-nucleotide 
RNA strands with four different nucleotides $A, U, G$ and $C$. Assume that a small fraction of the $4^{100}$ different possible sequences accomplishes a specified function $x$ to a significant degree (corresponding to a relatively high functional information). A significantly greater number of configurations will be somewhat less efficient in accomplishing function $x$ (corresponding to a smaller value of functional information), and the majority of configurations will display little or no function (Lenski et al. 2003; Carothers et al. 2004).

We define "degree of function" $\left(E_{x}\right)$ as a quantitative measure of a configuration's ability to perform the function $x$. In an enzyme, for example, $E_{x}$ might be defined as the increase in a specific reaction rate that is achieved by the enzyme, whereas in a system with water flowing over sand ripples, $E_{x}$ might be defined as the rate at which energy dissipates by turbulence, compared to water flowing over a smooth, unpatterned sand surface. The scale or units of $E_{x}$ may be chosen somewhat arbitrarily and will depend on the character of the function. For example, the catalytic efficiency of an enzyme might be measured as percentage rate enhancement, or it might be recorded as a decreased activation energy (which is proportional to the log of rate enhancement).

We calculate functional information $\left[/\left(E_{x}\right)\right]$ in terms of a specified degree of function $\left(E_{x}\right)$. In most cases, a minute fraction [ $\left.F\left(E_{x}\right)\right]$ of all possible configurations of a system achieves a degree of function $\geq E_{X}$. Thus, functional information (in bits) is defined in terms of $F\left(\mathscr{E}_{X}\right)$ :

$$
/\left(E_{x}\right)=-\log _{2}\left[F\left(E_{x}\right)\right]
$$

In a system with $N$ possible configurations (e.g., a sequence of $n$ RNA nucleotides with $N=4^{\mathrm{n}}$ different sequences, and assuming equal probability for all sequences):

$$
/\left(E_{x}\right)=-\log _{2}\left[M\left(E_{x}\right) / N\right]
$$

where $M\left(E_{\chi}\right)$ equals the number of different RNA configurations with degree of function $\geq E_{X}$ Note that the fraction of configurations, $F\left(E_{x}\right)$, capable of achieving a specified degree of function will generally decrease with increasing $E_{x}$ (Szostak 2003).

This simple formalism leads to several important consequences. First, the greatest possible functional information for a given system occurs in the case of a single configuration that displays the highest possible degree of function, $E_{\text {max }}$ :

$$
/\left(E_{\text {max }}\right)=-\log _{2}[1 / M]=\log _{2} N \text { (in bits). }
$$

This maximum functional information is thus equivalent to the maximum number of bits necessary and sufficient to specify any particular configuration of the system. Given such a maximally functional configuration, the only way to increase the function (and, by definition, the functional information) of the system is to increase to total number of different possible configurations, $N$, either (1) by increasing the number of interacting agents, (2) by increasing the diversity of interacting agents, or (3) increasing the diversity of interactions among agents.

On the other hand, the minimum functional information of any system is zero. This situation obtains for configurations with the lowest degree of function, $E_{\text {min }}$, because all possible states have $E_{x} \geq E_{\text {min }}$ :

$$
/\left(E_{\text {min }}\right)=-\log _{2}(N / N)=-\log _{2}(1)=0 \text { bits }
$$

Thus, functional information must increase with degree of function, from zero for no function (or minimal function) to a maximum value corresponding to the number of bits that are both necessary and sufficient to specify any system configuration.

It is important to note that functional information is defined only in the context of a specific function $x$. For example, the functional information of a ribozyme will be greater than zero with respect to the catalysis of one specific reaction, but will be zero or minimal with respect to most other reactions. Functional information thus depends on both the system and on the specific function under consideration. Note also that if no configuration of a system is able to accomplish a specific function $x$ [i.e., $\left.M E_{x}\right)=0$ ], then the functional information corresponding to that functionis undefined, no matter how complex the patterning of that state appears to be.

\section{Function versus information}

Functional information is a statistical property of a system of many different agent configurations (e.g., RNA nucleotides, neurons, ants) with respect to a specific function. In order to quantify the functional information of any given configuration, we need to know the distribution of function for all possible configurations in the system, as well as the degree of function for that specific configuration. The distribution of function must be determined from the statistical properties of the system as a whole, as opposed to the intrinsic patterning of any one particular configuration. Analysis of the functional information of a specific configuration, therefore, requires a deep understanding of the system's agents, their various interactions, and the consequent diversity of configurations.

Comprehensive functional information analysis is difficult for most complex systems because of the combinatorial richness of configuration space. A large number of randomly generated configurations and their functions must be analyzed in order to obtain a statistically meaningful sampling of configuration space. Such analysis is currently impractical for many biological systems, but general trends have been determined for two cases. For example, Carothers et al. (2004) analyzed GTP-binding for randomly generated RNA aptamers and found that a 10-fold increase in GTP binding strength requires approximately 10 additional bits of information (i.e., specification of 5 additional nucleotides). They also recognized several distinct classes of RNA aptamers, which represent topologically different solutions to the problem of GTP

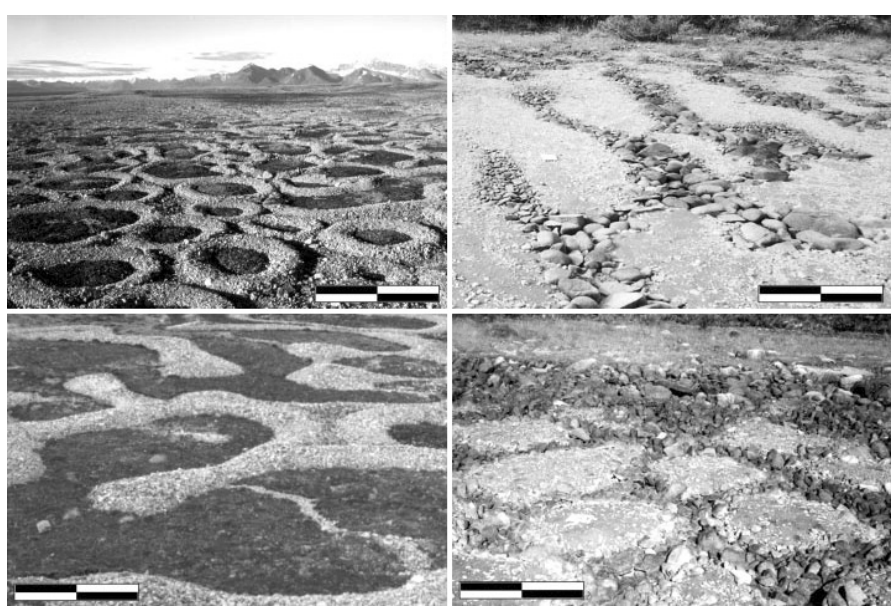

Fig. 5. "Patterned ground" with morphologies including circles, bifurcating lines, vermiform mounds and polygons, arises in Arctic regions, where cyclic freezing and thawing differentially repositions sediments of different sizes (from Kessler and Werner 2003). Photographs courtesy of B. T. Werner. 

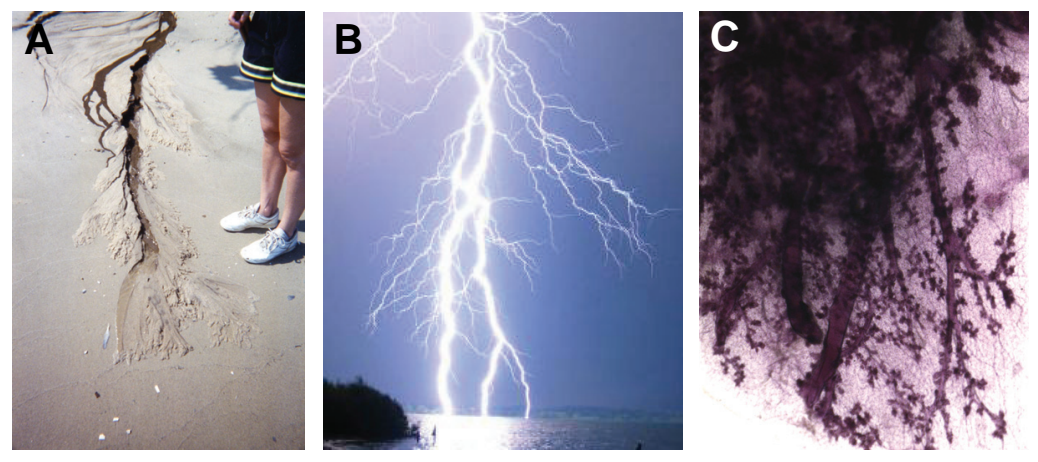

Adami 2003). Our formalism supports this conjecture but with an important caveat. Given our operational definition, any evolutionary mechanism that selects from a population of states based on enhanced function must lead to increased functional information with respect to the selected function. However, at the same time other functions that are not under selective pressure may remain constant or decrease in their degree of function.

Note in this regard that many natural and artificial systems are subject to cycles, such as wetting and drying, freezing and thawing, or day and night. Such cycles have the potential to apply repeated selective pressure on a system and thus accelerate evolution to states of increased degree of function and patterning (e.g., Hansen et al. 2001; Kessler and Werner 2003; see Fig. 5). Such cycling is thought to have played especially important roles in life's origins, for example in the selection, concentration and organization of life's molecular building blocks (Lahav et al. 1978; Orgel 1998; Hazen et

Fig. 6. Branching patterns arise in numerous non-living and living systems. (A) Sand patterning at the mouth of a small stream (photo courtesy of R. Hazen). (B) Lightning strike on a lake (photo courtesy of Google Images). (C) Vasculature in human mammary gland (photo courtesy of J.A. Mayer). Similar branching patterns are observed in raiding pathways of army ants, vein patterns in leaves, and the circulatory systems of vertebrates (Camazine et al., 2001).

binding.

In a similar effort, Hazen et al. (2007) investigated randomly generated Avida artificial life organisms, which consist of circular "genomes" of machine instructions. A small fraction of all genomes has the ability to perform one or more simple arithmetic of logic functions. They found a distinctive stepped relationship between the degree of function (measured as the number of times a given operation was performed by the Avida genome) and functional information. This result also pointed to the likely existence of several functional "islands" in configuration space multiple solutions to the problem of function.

In the future we hope to employ Monte Carlo procedures and genetic algorithms (e.g., Oganov and Glass 2006; Oganov and Valle 2009) to study other emergent systems, for example computational functions with electronic networks (Koza et al., 1997) and crystal structure prediction (Oganov et al., 2006). A similar approach might be applied to pattern formation in sand under the influence of flowing water (Bagnold 1988); the objective will be to generate numerous random, gravitationally stable sand configurations and calculate the efficiency of each configuration in dissipating energy or disrupting laminar flow. Similar techniques might be applied to the formation of chemical or cellular patterning under diffusive gradients.

\section{Functional information and the emergence of biologi- cal patterning}

Evolution is a process by which functional configurations are preferentially selected, while nonfunctional configurations are winnowed out. Functional patterns, including biological forms, thus arise through natural selective processes. The concept of functional information, coupled with principles of biological evolution, may shed light on the emergence of such patterns.

\section{The Law of Increasing Complexity}

The functional information formalism leads to intuitively reasonable conclusions with respect to the evolution of complex systems. Previous authors have proposed a "law of increasing complexity," which states that natural selection, acting alone, tends to increase the complexity of a system (McShea 2000; al. 2001).

The role of cycling is also illustrated in the molecular evolution experiments of Szostak and coworkers (Ellington and Szostak 1990; Wilson and Szostak 1999). Their RNA aptamer evolution experiments begin with large populations (as many as $10^{16}$ random sequences), which are subjected to a selective environment - for example, a target molecule. The small fraction of the random RNA population that selectively binds to the target can be isolated, amplified with mutations, and passed through the selection process. Repeated cycling rapidly reduces the combinatorially large number of possible configurations to a few highly functional RNA strands.

\section{Length selection pressure}

A system with several functions that is subjected to selective pressure for only one of those functions may display a substantial reduction in the size of the optimal system, especially in the face of limited resources or circumstances where rapid replication confers an advantage. This phenomenon of "length selection pressure" (e.g., Wang and Unrau 2005) was dramatically demonstrated in the original molecular evolution experiments of Spiegelman and colleagues (Mills et al. 1967), who observed an $83 \%$ reduction in $Q \beta$ viral genome length when selecting for rapid replication only. Similar instances of genome reduction are observed in many parasites and endosymbionts (Andersson and Andersson 1999; Shigenobou et al. 2000; Nakabachi et al. 2006).

\section{Inherent complexity of functions}

The functional information formalism points to the fact that some functions are inherently more complex than others. Some ribozymes, for example, require sequence lengths of several dozen nucleotides to display any significant degree of function. If, however, a ribozyme achieves a high degree of function with, for example, a sequence of only 15 nucleotides, then the functional information cannot exceed $/ \max \leq-\log _{2}\left(1 / 4^{15}\right)=30$ bits. Thus, some problems may have inherently simple solutions. In the context of life's origin, this observation raises the possibility that the sequence of some key macromolecules may be deterministic, because all possible short sequences are likely to be present in a local population. 
In this regard, any system subject to selective pressures will favor configurations exhibiting even a modest degree of function over non-functioning configurations. Consequently, simple systems with near-equilibrium configurations that possess the ability to perform some function are likely to serve as precursors to more complex, highly functional states. It is this type of process, for example, by which primitive amphiphilic vesicles that formed at equilibrium with the ability to encapsulate prebiotic molecules could have begun the evolution toward the complex cell membranes of contemporary biology.

\section{Evolution potential}

An important consideration in analyzing complex systems is the degree to which they can continue to evolve - what has been called "potential complexity" or "future complexity" as a measure of how much more complex an evolving system might become (e.g., Gell-Mann and Lloyd 2003). Functional information has the potential to shed light on this problem. If we are able to analyze a statistically significant fraction of a system's states and thus identify the relationship between $/\left(E_{x}\right)$ and $E_{x}$ then we should be able to deduce how close we are to $E_{\max }$ [and $/\left(E_{\max }\right)$ ]. We can then estimate the utility (or futility) of further evolutionary pressure, or perhaps enhance system parameters (i.e., the number of agents, the variety of agents, or the interconnectivity of agents) that will increase the inherent complexity of the system.

\section{Conclusions}

Studies of patterned systems, whether symbolic or physical, nonliving or living, reveal that complex forms, functions and behaviors emerge from the collective interactions of numerous agents, each of which responds to its own environment. In particular, patternings in developing biological systems arise from spatial and time-dependent interactions among cells and cell clusters. The functional information formalism not only provides a method for estimating the information required to achieve a particular degree of function, but it also points to factors that may govern the evolution to modified states.

\section{Acknowledgements}

I thank Prof. Cheng-Ming Chuong for his efforts in organizing this Special Issue of the Int. J. Dev. Biol. This research was supported by the NASA Astrobiology Institute, the National Science Foundation (USA), and the Carnegie Institution of Washington.

\section{References}

ADAMI, C. (1995). Learning and complexity in genetic auto-adaptive systems. Physica D 80: 154-170.

ADAMI, C. (2003). Sequence complexity in Darwinian evolution. Complexity 8: 4956.

ALLEN, C., BEKOFF, M. and LAUDER, G. (Eds.) (1998). Nature's Purposes. Analyses of Function and Design in Biology. MIT Press, Cambridge.

ANDERSSON, J.O. and ANDERSSON, S.G. (1999). Insights into the evolutionary process of genome degradation. Curr Opin Genet Dev 9: 664-671.

ARRAYAS, M., EBERT, U. and HUNDSDORFER, W. (2002). Spontaneous branching of anode-directed streamers between planar electrodes. Phys Rev Let 88: 174502.

AVERY, J. (2003). Information Theory and Evolution. World Scientific, Singapore. AYALA, F.J. (1999). Adaptation and novelty: Teleological explanations in evolution- ary biology. Hist Phil Life Sci21: 3-33.

BAGNOLD, R.A. (1988). The Physics of Sediment Transport by Wind and Water. American Society of Civil Engineers, New York.

BARLOW, G.W. (1974). Hexagonal territories. Animal Behav 22: 876-878.

BELL, G. (1997)." The Basics of Selection. Chapman and Hall, New York.

BEN-JACOB, E., SCHOCHET, O., TENENBAUM, A., COHEN, I., CZIROK, A. and VICSEK, T. (1994). Generic modeling of cooperative growth patterns in bacterial colonies. Nature 368: 46-49

BERNAL, J.D. (1951). The Origin of Life. Weidenfeld and Nicholson, London.

BERNSTEIN, M.P., SANDFORD, S.A., ALLAMANDOLA, L.J., GILLETTE, J.S., CLEMETT, S.J. and ZARE, R.N. (1999). UV irradiation of polycyclic aromatic hydrocarbons in ices: Production of alcohols, quinones, and ethers. Science 283: 1135-1138.

BERTALANFFY, L. (1968). General System Theory: Foundations, Applications, Development. G. Braziller, New York.

BORMAN, S. (2006). Chemical biology of the cell. Chem Eng News 84: 34-35.

CAMAZINE, S., DENEUBOURG, J.-L., FRANKS, N. R., SNEYD, H., THERAULAZ, G. and BONABEAU, E. (2001). Self-Organization in Biological Systems. Princeton University Press, Princeton, New Jersey.

CARLBERG, R. (1992). Galaxies, formation. In The Astronomy and Astrophysics Encyclopedia (Ed. S.P. Maran). Van Nostrand Reinhold, New York, pp. 268270.

CAROTHERS, J.M., OESTREICH, S.O., DAVIS, J.H. and SZOSTAK, J.W. (2004). Informational complexity and functional activity of RNA structures. J Am Chem Soc 126: 5130-5137.

CODY, G.D., BOCTOR, N.Z., BRANDES, J.A., FILLEY, T.R., HAZEN, R.M. and YODER, H.S. Jr. (2004). Assaying the catalytic potential of transition metal sulfides for prebiotic carbon fixation. Geochim Cosmochim Acta68:2185-2196.

DEACON, J.W. (2006). Fungal Biology, $4^{\text {th }}$ edition. Blackwell, Malden, Massachusetts.

DEAMER, D.W. and EVANS, J. (2006). Numerical analysis of biocomplexity. In Life As We Know lt. Cellular Origin, Life in Extreme Habitats and Astrobiology (Ed. J. Seckbach) Springer, New York, pp. 199-212.

DEAMER, D.W. and PASHLEY, R.M. (1989). Amphiphilic components of the Murchison carbonaceous chondrite: Surface properties and membrane formation. Origins Life Evol Biosphere 19: 21-38.

DE DUVE, C. (1995). Vital Dust: Life as a Cosmic Imperative. Basic Books, New York.

DE DUVE, C. (2005). The onset of selection. Nature 433: 581-582.

DENEUBOURG, J.-L., GOSS, S., FRANKS, N.R. and PASTEELS, J.M. (1989). The blind leading the blind: Modeling chemically mediated army ant raid patterns. $J$ Insect Behav 2: 719-772.

DOYLE, J. and M. CSETE (2007). Rules of engagement. Nature 446: 860.

DWORKIN, J.P., DEAMER, D.W., SANDFORD, S.A. and ALLAMANDOLA, L.J. (2001). Self-assembling amphiphilic molecules: Synthesis in simulated interstellar/precometary ices. Proc Nat/ Acad Sci USA 98: 815-819.

EDWARDS, W.S. and FAUVE, S. (1993). Parametrically excited quasicrystalline surface waves.Parametrically excited quasicrystalline surface waves. Phys Rev E 47: R788-R791.

EHRENFREUND, P. and CHARNLEY, S.B. (2000). Organic molecules in the interstellar medium, comets, and meteorites. Ann Rev Astron Astrophys 38 427-483.

ELLINGTON, A.E. and SZOSTAK, J.W. (1990). Selection in vitroof single-stranded DNA molecules that fold into specific ligand binding sites. Nature 346: 818-822.

EMANUEL, K. (2006). Hurricanes: Tempests in a greenhouse. Phys Today 59: 7475.

FEDDER, T. (2007). Statistical physics is for the birds. Phys Today 60: 28-30.

FERRIS, J.P. (1999). Prebiotic synthesis on minerals: Bridging the prebiotic and RNA worlds. Biol Bull 196: 311-314.

FIELD, R. and BURGER, M. (Eds. ) (1981). Oscillations and Travelling Waves in Chemical Systems. Wiley, New York.

FRANKS, N.R. andnDENEUBOURG, J. L. (1997). Self-organizing nest construction in ants: Individual worker behaviour and the nest's dynamics. Animal Behav 
54: 779-796.

FRANKS, N.R., WILBY, W., SILVERMAN, B.W. and TUFTS, C. (1992). Selforganizing nest construction in ants: sophisticated building by blind bulldozing. Animal Behav 44: 357-375.

FRISCH, M. (1995). Turbulence: The Legacy of A. N. Kolmogorov. Cambridge University Press, Cambridge.

GELL-MANN, M. (1995). What is complexity? Complexity 1: 16-19.

GELL-MANN, M. and LLOYD, S. (2003). Effective complexity. In Nonextensive Entropy: Interdisciplinary Applications (Eds. M. Gell-Mann and C. Tsallis). Oxford University Press, New York, pp.387-389.

GILBERT, W. (1986). The RNA world. Nature 319: 618.

HANSEN, J.L., VAN HECKE, M., HANNING, A., ELLEGAARD, C., ANDERSEN, K.H., BOHR, T. and SAMS, T. (2001). Instabilities in sand ripples. Nature 410: 324.

HAZEN, R.M. (2005). Genesis: The Scientific Quest for Life's Origins. Joseph Henry Press, Washington, DC

HAZEN, R.M. (2006). Mineral surfaces and the prebiotic selection and organization of biomolecules. Am Minera/91: 1715-1729.

HAZEN, R.M. and DEAMER, D.W. (2007). Hydrothermal reactions of pyruvic acid: Synthesis, selection, and self-assembly of amphiphilic molecules. Origins Life Evol Biosphere 37: 143-152.

HAZEN, R.M., FILLEY, T. and GOODFRIEND, G.A. (2001). Selective adsorption of $\mathrm{L}$ - and D-amino acids on calcite: Implications for biochemical homochirality. Proc Natl Acad Sci USA 98: 5487-5490.

HAZEN, R.M., GRIFFIN, P., CAROTHERS, J. and SZOSTAK, J. (2007). Functional information and the emergence of biocomplexity. Proc Nat/ Acad Sci USA 104: 8574-8581

HEINEN, W. and LAUWERS, A.M. (1996). Organic sulfur compounds resulting from interaction of iron sulfide, hydrogen sulfide and carbon dioxide in an aerobic aqueous environment. Origins Life Evol Biosphere 26: 131-150.

HÖFER, T., SHERRATT, J.A. and MAINI, P.K. (1995). Dictyolstelium discoideum. cellular self-organization in an exciteable biological medium. Proc Royal Soc London B 259: 249-257.

HOLLAND, J.H. (1995). Hidden Order. Helix Books, Reading, Massachusetts.

HOYLE, R. (2006). Pattern Formation: An Introduction to Methods. Cambridge University Press, New York.

JUNG, H.-S., FRANCIS-WEST, P.H., WIDELITZ, R.B., JIANG, T.-X., TINGBARRETH, S., TICKLE, C., WOLPERT, L. and CHUONG, C.-M. (1998). Local inhibitory action of BMPs and their relationships with activators in feather formation: Implications for periodic patterning. Dev Bio/196: 11-23.

KÅHRE, J. (2002). The Mathematical Theory of Information. Kluwer, Boston.

KAUFFMAN, S. A. (1993). The Origins of Order: Self-Organization and Selection in Evolution. Oxford University Press, New York.

KESSLER, M.A. and WERNER, B.T. (2003). Self-organization of sorted patterned ground. Science 299: 380-383.

KO, E.P., YOMO, T. and URABE, I. (1994). Dynamic clustering of bacterial population. Physica D75: 81-88

$\mathrm{KOCH}$, A.J. and MEINHARDT, H. (1994). Biological pattern formation: from basic mechanisms to complex structures. Rev Mod Phys 66: 1481-1507.

KOSCHMEIDER, E.L. (1981). Bénard convection. In Order and Fluctuations in Equilibrium and Nonequilibrium Statistical Mechanics (Eds. G. Nicolis, G. Dewel and J.W. Turner). Wiley, New York.

KOZA, J.R., BENNETT, F.H. III, LOHN, J.D., DUNLAP, F., ANDRE, D. and KEANNE, M.A. (1997). Automated synthesis of computational circuits using genetic programming. In Proceedings 1997 IEEE International Conference on Evolutionary Computation. IEEE Press, Piscataway, New Jersey, pp. 447-452.

LAHAV, N., WHITE, D. and CHANG, S. (1978). Peptide formation in the prebiotic era: Thermal condensation of glycine in fluctuating clay environments. Science 201: 67-69

LAWRENCE, P.A. (1992). The Making of a Fly: The Genetics of Animal Design. Blackwell Scientific, Oxford.

LECHLEITER, J.D., GIRARD, S., PERALTA, E. and CLAPHAM, D. (1991). Sprial calcium wave propagation and annihilation. Science 252: 123-126.

LENSKI, R.E., OFRIA, C., PENNOCK, R.T. and ADAMI, C. (2003). The evolution- ary origin of complex features. Nature 423: 139-144.

MAINI, P.K., BAKER, R.E. and CHUONG, C.-M. (2006). The Turing model comes of age. Science 314: 1397-1398.

MCSHEA, D.W. (2000). Functional complexity in organisms: Parts as proxies. Biol Phil 15: 641-668.

MEINHARDT, H. (1995). The Algorithmic Beauty of Sea Shells. Springer, Berlin.

METZGER, R. and KRASNOW, M.A. (1999). Genetic control of branching morphogenesis. Science 284: 1635-1639.

MILLER, S.L. (1953). A Production of amino acids under possible primitive Earth conditions. Science 117: 528-529.

MILLER, S.L. and UREY, H.C. (1959). Organic compound synthesis on the primitive Earth. Science 130: 245-251.

MILLS, D. R., PETERSON, R.L. and SPIEGELMAN, S. (1967). An extracellular Darwinian experiment with a self-duplicating nucleic acid molecule. Proc Nat/ Acad Sci USA 58: 217-224

MOROWITZ, H.J. (1982). Foundations of Bioenergetics. Academic Press, New York.

MOROWITZ, H.J. (1992). The Beginnings of Cellular Life: Metabolism Recapitulates Biogenesis. Yale University Press, New Haven.

MOROWITZ, H.J. (2002). The Emergence of Everything. Oxford University Press, New York.

MURRAY, J.D. (1981). A pre-pattern formation mechanism for animal coat markings. J Theor Bio/88: 161-199.

NAKABUCHI, A., YAMASHITA, A., TOH, H., ISHIKAWA, H., DUNBAR, H.E., MORAN, N.A. and HATTORI, M. (2006). The 160-kilobase genome of the bacterial endosymbiont Carsonella. Science 314: 267.

NETTESHEIM, S., VON OERTZEN, A., ROTERMUND, H.H. and ERTL, G. (1993). Reaction diffusion patterns in the catalytic CO-oxidation on $\mathrm{Pt}(110)$ : front propagation and spiral waves. J Chem Phys 98: 9977-9985.

NICOLIS, G. and PRIGOGINE, I. (1989). Exploring Complexity. W. H. Freeman, New York.

NÜSSLEIN-VOLHARD, C. (2006). Coming to Life: How Genes Drive Development. Kales Press, Carlsbad, California.

OGANOV, A.R. and GLASS, C.W. (2006). Crystal structure prediction using $a b$ initio evolutionary algorithms: principles and applications. J Chem Phys 124: 244704.

OGANOV, A.R. and VALLE, M. (2009). How to quantify energy landscapes of solids. J Chem Phys 130: 104504

OGANOV, A.R., GLASS, C.W. and ONO, S. (2006). High-pressure phases of $\mathrm{CaCO}_{3}$ : crystal structure prediction and experiment. Earth Planet Sci Let 241: 95-103.

OUYANG, Q. and SWINNEY, H.L. (1991). Transition from a uniform state to hexagonal and striped Turing patterns. Nature 352: 610-612.

ORGEL, L.E. (1986). RNA catalysis and the origin of life. J Theor Bio/123: 127149.ORGEL, L.E. (1998). Polymerization on the rocks: Theoretical introduction. Origins Life Evol Biosphere 28: 227-234.

PALMEIRIN, I., HENRIQUE, D., ISH-HOROWITCZ, D. and POURQUIÉ, O. (1998). Avian hairy gene expression identifies a molecular clock linked to vertebrate segmentation and somitogenesis. Ce//91: 639-648.

PARRISH, J.K. and HAMMER, W.M. (1997). Animal Groups in Three Dimensions: How Species Aggregate. Cambridge University Press, Cambridge.

PARK, C.F. Jr. and MACDIERMID, R.A. (1964). Ore Deposits. Freeman, San Francisco.

PETROV, V., OUYANG, Q. and SWINNEY, H.L. (1997). Resonant pattern formation in a chemical system. Nature 388: 655-657.

PETTIJOHN, F.J. (1957). Sedimentary Rocks, $2^{\text {nd }}$ Edition. Harper \& Row, New York.

PIEKNY, A.J. and MAINS, P.E. (2003). Squeezing an egg into a worm: C. elegans embryonic morphogenesis. Sci World J3: 1370-1381.

SAGAN, C. (1961). On the origin and planetary distribution of life. Radiation Res 15: $174-192$

SHECHTMAN, D., BLECH, I., GRATIAS, D. and CAHN, J.W. (1984) Metallic phase with long-range orientational order and no translational symmetry. Phys Rev Let 
53: 1951-1953

SHALIZI, C.R. (2006). Methods and techniques of complex systems science: An overview. Web-based publication: http://arxiv.org/PS_cache/nlin/pdf/0307/ 0307015.pdf

SHIGENOBU, S., WATANABE, H., HATTORI, M., SAKAKI, Y. and ISHIKAWA, H. (2000). Genome sequence of the endocellular bacterial symbiont of aphids Buchnera sp. Nature 407: 81-86.

SICK, S., REINKER, S., TIMMER, J. and SCHLAKE, T. (2006). Wht and Dkk determine hair follicle spacing through a reaction-diffusion mechanism. Science 314: 1447.

SOLÉ, R. and GOODWIN, B. (2000). Signs of Life: How Complexity Pervades Biology. Basic Books, New York.

SOLÉ, R.V., BASCOMPTE, J. and VALLIS, J. (1992). Stability and complexity in spatially-extended two-species competition. J Theor Biol 159: 469-476.

SWENSON, R. and TURVEY, M.T. (1991). Thermodynamic reasons for perception-action cycles. Ecol Psych 3: 317-348.

SWINNEY, H.L. (2000). Emergence and evolution of patterns. In Stochastic Dynamics and Pattern Formation in Biological and Complex Systems (Eds. S. Kim, K.J. Lee and W. Sung). American Institute of Physics, New York, pp. 3-22.

SZOSTAK, J. (2003). Functional information. Nature 423: 689.

TURING, A.M. (1952). The chemical basis of morphogenesis. Phil Trans Royal Soc
B237: 37-72.

VIDAL, C. and PACAULT, A. (Eds.) (1984). Nonequilibrium Dynamics in Chemical Systems. Springer, Berlin.

VON BAEYER, H.C. (2003). Information: The New Language of Science. Weidenfeld \& Nicolson, London.

WANG, Q.S. and UNRAU, P.J. (2005). Ribozyme motif structure mapping using random recombination and selection. RNA 11: 404-411.

WIDELITZ, R.B., VELTMAAT, J.M., MAYER, J.A., FOLEY, J. and CHUONG, C.-M (2007). Mammary glands and feathers: Comparing two skin appendages which help define novel classes during vertebrate evolution. Seminars Cel/ Dev Biol 18: $255-266$

WILSON, D.S. and SZOSTAK, J.W. (1999). In vitro selection of functional nucleic acids. Ann Rev Biochem 68: 611-647.

WILSON, E.O. and HÖLLDOBLER, B. (1988). Dense heterarchies and mass communication as the basis of organization in ant colonies. Trends Ecol Evo/3: 65-68.

WOLFRAM, S. (2002). A New Kind of Science. Wolfram Media, Inc., Champaign, Illinois.

WU, P., JIANG, T.-X., SUKSAWEANG, S., WIDELITZ, R.B. and CHUONG, C.-M. (2004). Molecular shaping of the beak. Science 305: 1465-1466.

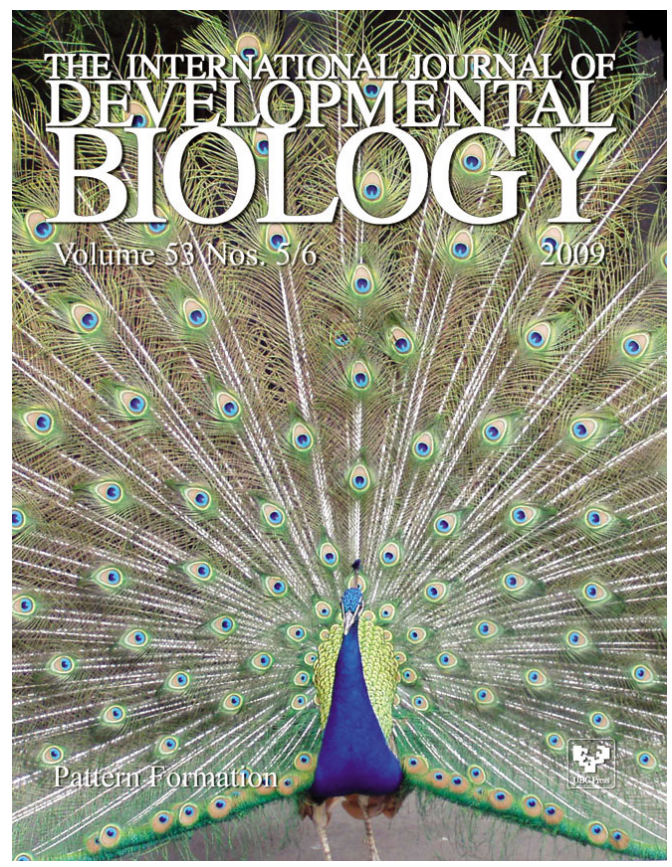

5 yr ISI Impact Factor $(2008)=3.271$

For all the latest on Pattern Formation research, see our latest Special Issue edited by C.-M. Chuong and M.K. Richardson. 


\section{Further Related Reading, published previously in the Int. J. Dev. Biol.}

See our Special Issue Developmental Morphodynamics edited by Lev Beloussov and Richard Gordon at: http://www.ijdb.ehu.es/web/contents.php?vol=50\&issue=2-3

See our Special Issue Evolution \& Development edited by Jaume Baguñà and Jordi García-Fernàndez at: http://www.ijdb.ehu.es/web/contents.php?vol=47\&issue=7-8

Differences in embryonic pattern formation between Caenorhabditis elegans and its close parthenogenetic relative Diploscapter coronatus

Vera Lahl, Jens Schulze and Einhard Schierenberg

Int. J. Dev. Biol. 53: doi: 10.1387/ijdb.082718vl (in press )

Pattern formation in the Drosophila eye disc Jean-Yves Roignant and Jessica E. Treisman Int. J. Dev. Biol. 53: doi: 10.1387/ijdb.072483jr (in press)

Principles of branch formation and branch patterning in Hydrozoa Stefan Berking

Int. J. Dev. Biol. (2006) 50: 123-134

Integument pattern formation involves genetic and epigenetic controls: feather arrays simulated by digital hormone models.

Ting-Xin Jiang, Randall B Widelitz, Wei-Min Shen, Peter Will, Da-Yu Wu, Chih-Min Lin, Han-Sung Jung and Cheng-Ming Chuong

Int. J. Dev. Biol. (2004) 48: 117-135

Patterning a multi-headed mutant in Hydractinia: enhancement of head formation and its phenotypic normalization.

Werner A Müller, Regina Teo and Frank Möhrlen

Int. J. Dev. Biol. (2004) 48: 9-15

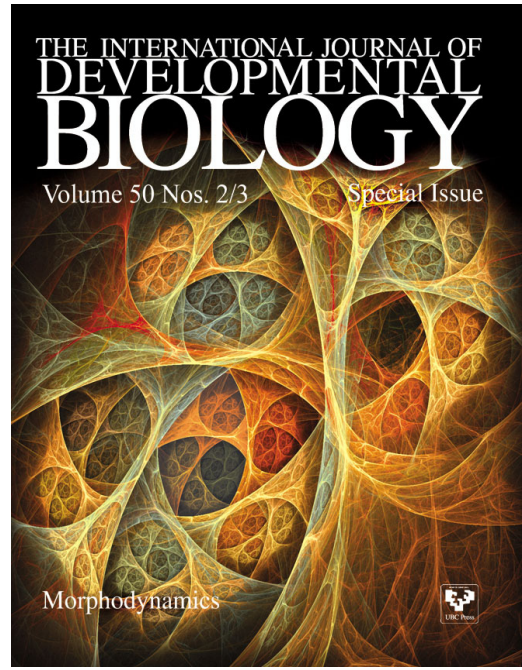

5 yr ISI Impact Factor $(2008)=3.271$

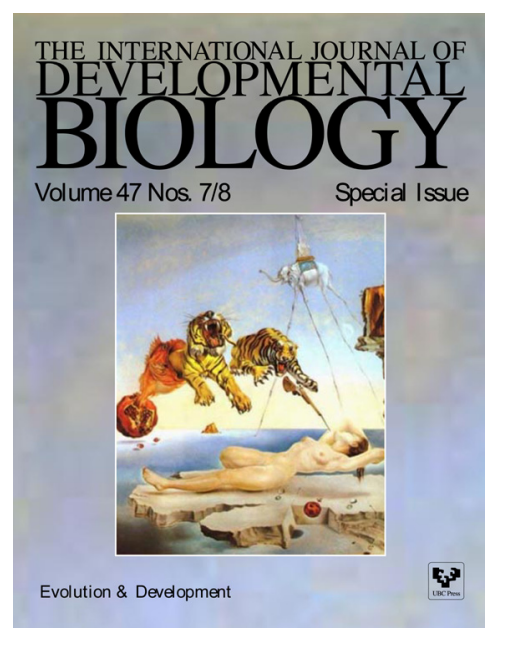

